\title{
Singular Space
}

\author{
Aleksandr Sevastjanov, PhD \\ Tallinn Technical University, Estonia
}

doi: 10.19044/esj.2016.v13n3p7 URL:http://dx.doi.org/10.19044/esj.2016.v13n3p7

\begin{abstract}
Classical physics is a bastion of order and stability, but when creating physics of unstable systems, we are faced with selecting one of many possibilities and limited predictability at all levels of observation. This fact forces us to consider the concepts of instability and chaos in physics, and to describe them by means of singular space.
\end{abstract}

Keywords: Singular space, Large Poincare Systems, uncertainty and reversibility

\section{Introduction}

In this paper, we introduce the concept of singular $(S g)$ space to describe quantum systems.

Idea of $S g$ - space consists that it is impossible to isolate a single point, as well as a separate element in this space, therefore, the dimension of this space (such as a line, plane, etc.) is not determinable, and therefore it has a quality of uncertainty, but brings the order to the understanding of the quantum world, which criterions are uncertainty and reversibility I. Prigogine et al., (2003). The historical excursion into the problems of studying the microcosm is being made because of the necessity of introducing this concept.

It is assumed that all physical objects are quantum systems, and there is often a problem of uncertainty and reversibility in the description of physical processes. The values of these events may be indefinite, in this work it is shown in the various examples.

It states a significant problem in the understanding of the classical laws of physics, written for such situations in the macrocosm, when the proportion of uncertainty is small and has little effect on getting of the desired value, in microcosm this proportion becomes significant, and the use of the classical laws gives probability results, the use of the $S g$ - space removes conflict between macro and microcosm. 
$\mathrm{Sg}$ - space as a mandatory attribute of a quantum system is a new concept introduced to describe quantum systems and their conduct.

The aim of this work is to make an overview of the problems associated with the use of the classical laws of physics to the microworld and showing the relevance of the concept of $\mathrm{Sg}$ - space for their solution.

\section{Singular space and its relevance.}

The geometric point has no measuring characteristics. But it is possible to give it some internal contents that can't be expressed by any geometry. Then an indivisible $S g$ space is formed as equivalent to a geometric point at which it is impossible to single out any particular part. $\mathrm{Sg}$ - point surrounding space (environment) is the inverse of $S g$ - point, and it also has the indivisible inner state.

$\mathrm{Sg}$ - space and its (orthogonal by definition) environment $\left(\mathrm{Sg}^{-1}\right.$ space) comprise an oscillating pair, where they functionally periodically replace each other (the oscillating symmetry is formed), this pair can be defined as oscillating single entity - $S g$ - oscillator. $S g$ - oscillator can be symbolically compared with the model of pendulum, where traditional springs and weights are replaced by the proposed pair. It allows you to build an oscillating system "on the empty place” in the absence of material objects.

Resulting action of $\mathrm{Sg}$ - oscillator for the period is zero, and hence incomparable and can't be detected. But as part of the quantum system $S g$ oscillator is a source of uncertainty. The interaction of such system with other systems leads to the production of real results that meet the normal probability distribution. The pendulum symbolizing $S g$ - oscillator surely has the maximum value, which when exceeded leads to disruption of its symmetry and the resulting effect for the period becomes different from zero, the space becomes a real and measurable.

In geometry the space is considered to be static and unlimited, in physics - and dynamic and limited, dynamism implies a certain speed, which has a limit. Therefore $S g$ - oscillator is limited. Measurable characteristics of such an oscillator are undefined, but as a result of interaction with other quantum systems it is expressed with specific rational values. Thus, the real space is determined by rational discrete values separated by $S g$ - intervals. They are small enough for macrocosm to consider the real space smooth.

Such $S g$ - oscillators are in the annex to the Schrodinger equation wave function, in general, the Large Poincare Systems (LPS) V.I. Arnold (2014) that are discussed in this paper below. 
The physical processes occurring in $S g$ - space are reversible. It is impossible to determine the priority of any of its parts, what is the reason and what is the result of the oscillatory process. $S g$ - space exists out of time, out of energy.

Problems with $S g$ - space, were always appearing in the history of physics, and therefore there is historical review of these issues below.

Singular space is needed in order to define the energy of quantum systems.

\section{Singular function}

The singular function - as a special case of representation of $\mathrm{Sg}$ space - a continuous function, which value of the derivative is equal to zero almost everywhere U. A. Hramov (1983), Royden (1988). Sg - space has only one point at which the derivative is not zero, because it is represented by this point.

Minkowski singular function is one-to-one and maintaining order converts quadratic irrationality (i.e. numbers of the form $(a+\sqrt{b})$, where $a$ and $b$-rational) on the interval $[0 ; 1]$ to the rational numbers on the same interval, and the rational numbers - in dyadic-rational U. A. Hramov (1983).

Minkowski function - an attempt of conformal mapping of irrational space on rational one.

Dirac singular function ( $\delta$ - function) - is a singular generalized function. It allows you to record the spatial density of a physical quantity (mass, charge, intensity of the heat source, power and so on), concentrated and applied to a single point. $\delta$ - function is not a function in the classical sense. It is defined as a continuous linear functional on the space of differentiable functions U. A. Hramov (1983).

So, this function identifies the domain in which it is impossible to single out any particular point, and this area is served as a one common functional. If Paul Dirac gave this function oscillating character (i.e. opposed similar antipode to $\delta$ - function), he would get a function that describes the $\mathrm{Sg}$ - oscillator (what he actually wanted). 


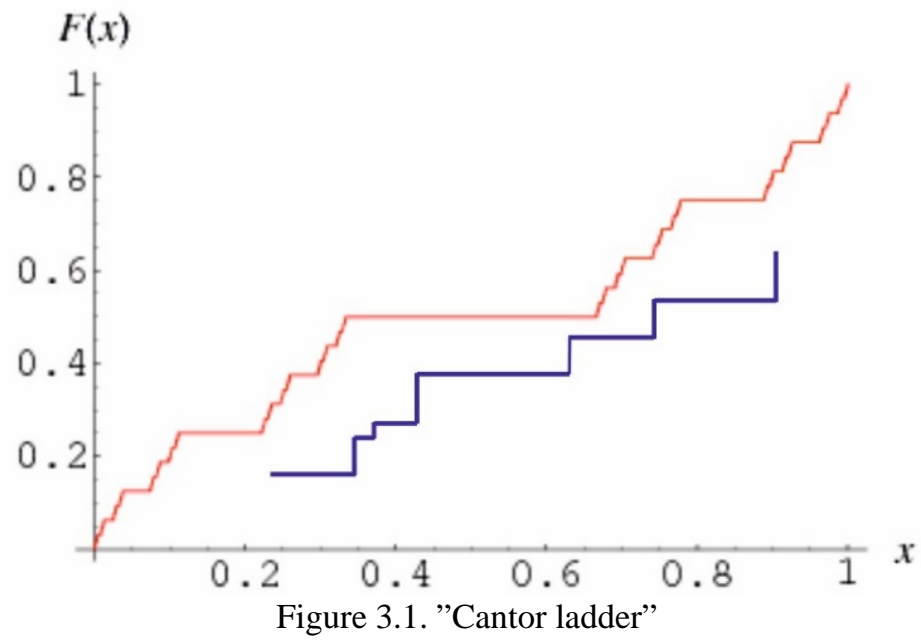

"Cantor ladder" is a special case of the Minkowski singular function.

Derivative of Cantor ladder is defined and equal to zero at all points except the Cantor plurality.

Cantor ladder is continuous, has bounded variation, but not absolutely continuous Bailey et al., (2002).

This way you can show quantum systems in transitions from one energy level to another, where the transition itself takes some time. But for $\mathrm{Sg}$ - presentation when the transition does not take time, inclined portions of red graph in Fig. 3.1 should be vertical as in the graph in blue.

Any application of singular functions rests on the problem of $S g$ space to justify such an analytical gap. Blue line in Fig. 3.1 satisfies such criteria as uncertainty and reversibility, imposed on quantum theory.

\section{Vector flow}

In the rational space would be sufficient for this purpose to use the product of a vector on the flow area. However, a single vector is not determined in this way - it is prevented by probability distribution of the flow, and the following Gauss theorem is the solution of the problem of determining the total flow and some part of it Alfred Gray et al., (2006).

The flow - an area which has some internal movement, which can't be shown by individual dots, otherwise the whole movement could be shown by one vector applied to the surface of an isotropic. Fig. 4.1 shows the symbolic vector flow, but from which it is clear that this flow can't be described by one vector, the flow is engulfed by chaotic influence, but some of its parts can be considered as linear or ordered to give it some uncertainity. 
Poynting vector - the energy flow density through the surface - also show the part of the energy of the oscillating process in the $\mathrm{Sg}$ - oscillator. The concept of the flow - a sign of $S g$ - space in which only a certain part is allowed for consideration.

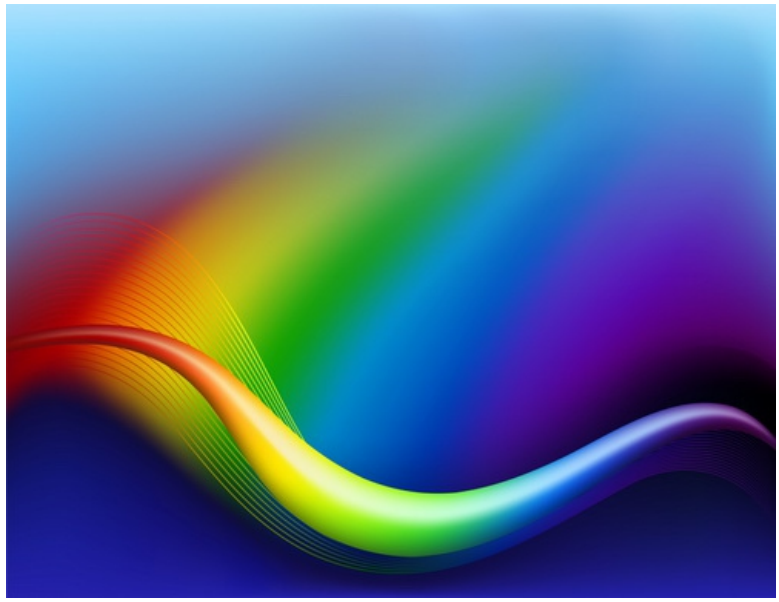

Figure 4.1. Vector flow

Flow of vector field through a surface - Gauss theorem - the surface integral of the second kind L. A. Bessonov (2016):

$$
\Phi=\iint F d S, \quad F \text { - vector field. }
$$

This is a common form of representation of the vector flow.

Here in $S g$ - space separate area "is torn", which is given rational character.

In the first half of the 19th century, Carl Friedrich Gauss applied the method of converting the triple integral to the surface (along with $\mathrm{M}$. Ostrogradski), admiting the irrationality of one of the differentials. It was used in the solution of problems in electrodynamics. Therefore, the problem of representation of $\mathrm{Sg}$ - space has appeared during of development of the electrodynamics.

\section{Heisenberg's uncertainty}

Heisenberg uncertainty relations are a theoretical one, it is the limit of accuracy of simultaneous two non-commuting observable measurements. For example, according to the uncertainty principle, the particles can't simultaneously have precisely measured position and velocity (momentum) D. Sen (2014).

The uncertainty principle, founded by Werner Heisenberg in 1927, is one of the cornerstones of the physical quantum mechanics. 
It is a consequence of the principle of wave-particle duality A. S. Davydov (1973).

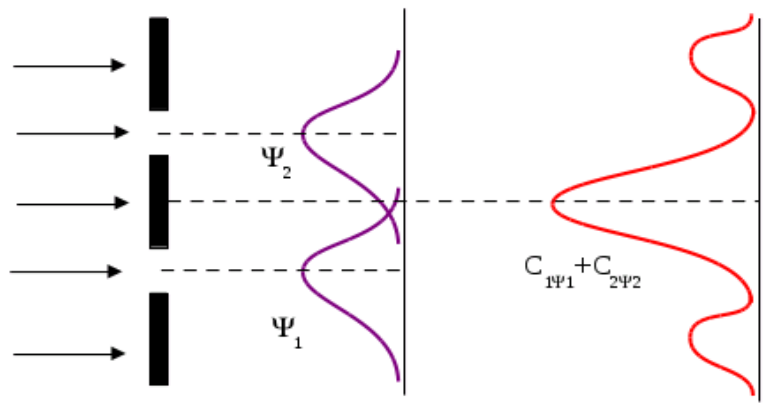

Figure 5.1. The distribution of photons in the interstitial space

This principle emphasizes the importance of chaos in quantum systems, their $\mathrm{Sg}$ - presence in each quantum system. This uncertainty pursues any physical process in a greater or lesser degree, and can show it quantum nature.

Any micro-object (in our case - a photon) passing through the slot detects the $S g$ - presence expressed by probability distribution (see Fig. 5.1), called the wave function $\Psi_{1}$ (and $\Psi_{2}$ ) and the density distribution of $C_{1 \Psi_{1}}$ (and $C_{1 \Psi 2}$ ). Thus the impression of the wave-particle duality is made, but any quantum system consists of rational and irrational parts. The last part gives the uncertainty $(\mathrm{Sg})$ to the system.

The same usage of $S g$ - space can be given to explain the nature of wave-particle duality.

With the introduction of the concept of $S g$ - space uncertainty becomes a physical concept.

\section{Bohr quantum chaotic system}

According to Niels Bohr measuring instrument must fulfill an essential function: it must be an intermediary between the laws of quantum mechanics, running on a microscopic and macroscopic level I. Prigogine et al., (2003). 


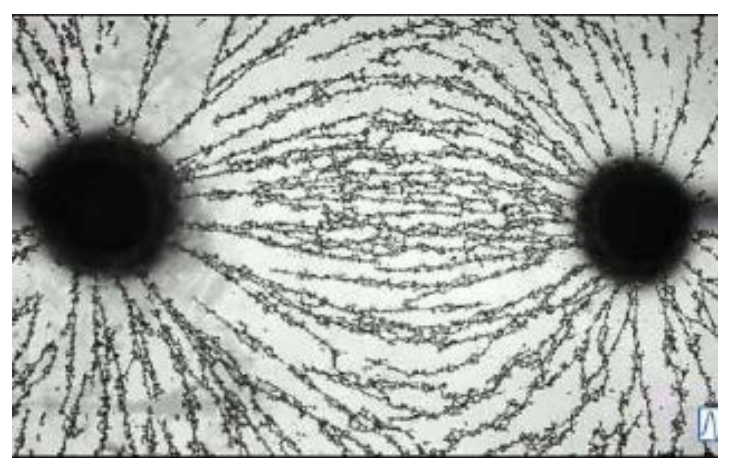

Figure 5.1. The outlines of the electric field

Quantum theory does not pass smoothly into the classical theory. The measuring device of Bohr "smoothens" the transition N. Bohr (1918). Bohr pointed out that it should be a dynamic quantum system, but did not indicate how to acquire it. The exact meaning to the Bohrs claims is given in the quantum theory of the LPS (Large Poincare Systems, regarded below in section "Poincare theorem"), demanding the measuring instrument to be the chaotic quantum system N. Bohr (1937). Bohr emphasized the possibility of such actions on the part of non-integrable system of the instrument, which turns it into integrable (quantum transition), as a result the system receives a specific real value, which is usually called the quantum.

It means that the conflict of two $\mathrm{Sg}$ - oscillators is needed for the assessment of a certain physical value. The measure is not produced without this conflict, because $S g$ - space does not accept real values.

In Bohr representation $\mathrm{Sg}$ - oscillator of the system is implied a "chaotic" quantum system, which is a reference for the measurements of $\mathrm{Sg}$ oscillators of other quantum systems.

Due to the conflict of two $\mathrm{Sg}$ - oscillators it is possible to see the outlines of the electric field. Field is not shown itself, but when the $\mathrm{Sg}$ oscillator of the electric charge came into conflict with similar oscillators (in our case - sawdust), they received an orientation, and the field became conspicuous. Fig. 6.1 illustrates how the field is taking shape. This effect makes the unimaginable physical processes visual, and we use it successfully.

\section{Schrodinger equation}

Quantum states described by the Schrodinger equation requires the collapse of the wave function, which is not part of the fundamental equation of quantum mechanics.

This equation is deterministic and reversible in time. Here the wave function determines the borders of the reversibility of processes in time, and it is $S g$. But the equation does not attribute the objective meaning to the 
events, it is the reverse side of determinism in the description of a microcosm where the quantum effects should be considered as an event. It is not provided in the equation, but it is necessary to have an opportunity to review the certainty and uncertainty, that take place in microcosm. It is provided by the Poincare theorem, which is regarded in the next section.

\section{Poincare theorem}

This theorem is about integrable and non-integrable dynamical systems I. Prigogine (1997).

Poincare question was formulated in terms of the Perturbation theory. It has started with the Hamiltonian written as the sum of $H_{0}(J)+\lambda(J, a) \mathrm{N}$. Hadyn et al., (2002). Free Hamiltonian $H_{0}(J)$ corresponds to the integrable system, isomorphic system of free particles. Then $H_{0}$ indignant interaction potential $\lambda(J, a)$, where $\lambda$ - the coupling constant (a parameter measuring the intensity of the interaction). The interaction potential makes a dynamic non-integrable system. The reason of nonintegrability - the existence of resonances between the degrees of freedom. Resonances are responsible for the inability to exclude interactions.

In this case, the resonances are a special case of $S g$ - space, which is not integrable and goes beyond the classical concepts.

Gibbs microcanonical distribution - the probability distribution of the different states in a closed macroscopic system, i.e. the systems, which do not interact with surrounding bodies and are non-dissipative D. Zubarev et al., (2002). Such a system in fact can't be obtained and it is idealized. Its states are degenerate: each energy value may correspond to different states of the system. This is a normal distribution within the non-integrable part of the system, and the distribution is a random process, which each time belongs to an individual quantum system.

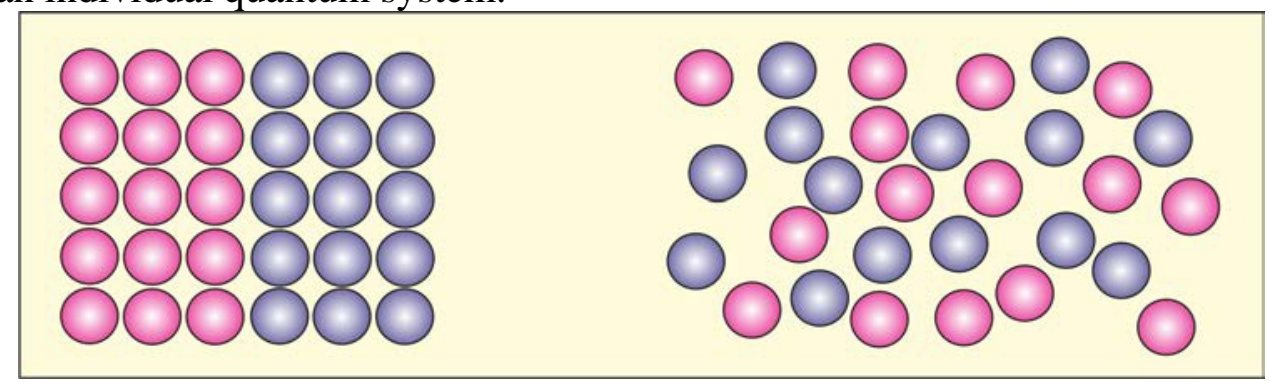

Figure 8.1 Order (left) and chaos (right)

Fig. 8.1 shows a classic view of the order (left) and chaos (right). Order can be described - integrate, chaos can't be described - it is non- 
integrate ( $\mathrm{Sg}$ ) Beekman (2009). The whole world is in between these two states. But this description is based on a symbolic representation of an ideal gas model. Both represented parts of the original order are involved in the chaos.

LPS mainly describe one element of an ideal chaos. Hence, this element is a quantum system, which consist of two parts, as it is shown in Fig. 8.2.

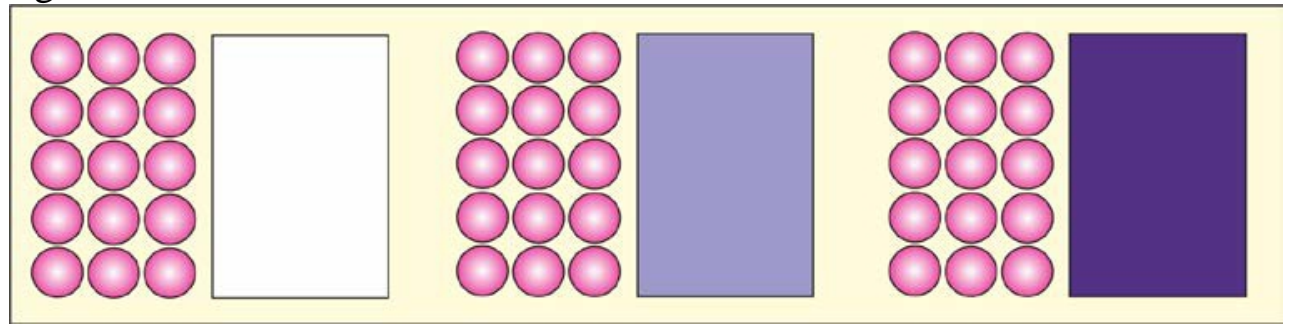

Figure 8.2 The Large Poincare Systems

Any physical object is related to the LPS to some extent, but this property becomes considerable and determinable in relation to the quantum systems. In case of non-dissipative systems chaos becomes a stable quantum state (but undeterminable). Integrable part (red) has the character of constant. Non-integrable (blue) part takes the value from zero to a maximum according to probabilistic nature of the Gibbs distribution (boundary conditions are on the left and the right, intermediate - in the center). In the quantum representation, non-integrable part does not consist of separate elements. It is an $S g$ - indivisible part, which takes a solid color in the Fig. 8.2. Only the $S g$ - part takes place in the chaos.

The multiplicity of degeneracy of the state is called the number of states, whenever there is a new quantum transition the $S g$ - space (oscillator) appears, which gives a "new life" to the quantum system.

Example of LPS: the expression of the photoelectric effect $E=h v+e U$ L.D. Landau et al., (1974). The first term expresses an integrable part of the energy, and the second - $S g$ - part, having a probabilistic nature. Photon which transfers the energy to the electron has a constant and probabilistic part, is a typical representative of the LPS. It is impossible to show the chaos inside the quantum system without the usage of $\mathrm{Sg}$ - space.

\section{The summary of excursion}

The problem of representation of $S g$ - space has its own story cited in this work as a few examples. 
$S g$ - space eliminates the problem of the reversibility of physical processes and gives an opportunity to define the time as a physical factor, determined by the number of implementations of $S g$ - space (events). Quantum theory has two features - uncertainty and reversibility Prigogine et al., (2003), introducing the concept of $S g$ - space is justified as satisfying both of these criteria. Theories that do not meet such criteria, can't be considered quantum.

$\mathrm{Sg}$ - space is a source of real space, which is taken for granted in the classical models, but without a source of which it is not possible to determine the energy (beyond the scope of this work), without this energy is determined only symbolically.

The $S g$ - character belongs to any quantum system, it is a characteristic feature. This is reflected in the assessment of the phenomena discussed above, and requires adjustments for its understanding. "Special" properties of these manifestations don 't require only introducing the concept of a $S g$ - space and $S g$ - oscillator, but also their reference to the structure of quantum systems, which allows to come to their organization and structure as a result.

$S g$ - space makes real space discrete and processes reversible. But a large number of quantum systems together allows us to consider the real space smooth and reversibility of process hardly noticeable.

$\mathrm{Sg}$ - space allows to obtain an oscillating function (row of discrete values separated by singular intervals), i.e. get such theory of oscillations, which will perform a quantitative and qualitative assessment of quantum systems and, consequently, the phenomena of the macrocosm that, in fact, is the main task of physics.

$S g$ - space regards the description not only of the microcosm, it may give the impression that uncertainties become relatively smaller as you move to the macrocosm. There is a place for large and even macroscopic quantum systems in nature, in which the role of the $S g$ - space becomes significant, it is necessary to consider the description of such systems in the entire universe. This concept is a step to understanding the nature of physical fields, and in particular the nature of electricity.

Thus, it can be considered justified to use the concept of space to describe quantum systems and their interactions.

\section{References:}

1. I. Prigogine, I. Stengers. (2003) Время. Хаос. Квант. М.: УРСС, 242c. 
2. V.I. Arnold. (2014) Poincare A. Selected Works in three volumes. T. 3. $-682 \mathrm{p}$.

3. U. A. Hramov. (1983) Минковский Герман (Minkowski Herman) Физики: Биографический справочник Под ред. А. И. Ахиезера. - Изд. 2-е, испр. и дополн. - М.: Наука, - 400с.

4. Royden, H.L (1988) Real Analysis, Prentice-Hall, Englewood Cliffs, New Jersey

5. Bailey, D. H. and Crandall, R. E. (2002) Random Generators and Normal Numbers. Exper. Math. 11, 527-546.

6. Alfred Gray, Elsa Abbena, Simon Salamon. (2006) Gray Gauss's Theorema Egregium. \$20.2 in Modern Differential Geometry of Curves and Surfaces. Chapman and Hall/CRC Textbook, 531 p.

7. L. A. Bessonov. (2016) Теоретические основы электротехники. M. "Юрайт", - 703с.

8. D. Sen. (2014) The uncertainty relations in quantum mechanics (PDF). Current Science 107 (2) 203-218 p.

9. A.S. Davydov. (1973) Квантовая механика - 2-е изд., испр. и перераб. - М.: Наука, - 704c.

10. N. Bohr. (1918) On the Quantum-Theory of Line-Spectra I. Acad. Copenhague 8 serie, fasc. 1.

11. N. Bohr. (1937) Transmutations of Atomic Nuclei. Science 86 (2225): 161-165 p.

12. I.Prigogine. (1997) The end of certainty. The free press. N-York, London, Toronto, Sidney, Singapore, 208c.

13. N. Hadyn, J. Luevano, G. Mantica, S. Vpienti. (2002) Multifractal properties of return time statistics Phys. Rev. Lett. - 88 p.

14. D.Zubarev. V.Morozov. G. Repke. (2002) Статистическая механика неравновесных процессов. Том 1 Перевод с англ., Москва, Изд-во Физико-математической литературы, - 432c.

15. Beekman Informatics. (2009) Lecture 9. Information paradoxes. MGU. - 23 p.

16. L.D. Landau E.M. Lifshits. (1974) Qvantum mechanics (nonrelativistic theory). M.: Nauka, $-752 \mathrm{p}$. 